\title{
Kulturwissenschaft
}

\author{
Dorota Masiakowska-Osses
}

Adam-Mickiewicz-Universität, Poznań

DOI: $10.19195 / 0435-5865.142 .22$

\section{Das „Eintrittsbillet“" in die deutsche Gesellschaft? Die nationalsozialistische Vergangenheit aus der deutsch-türkischen Perspektive}

\section{Deutsche Vergangenheit und die Einwanderungsgesellschaft}

Nach einem verzögerten Prozess der Abrechnung mit dem Nationalsozialismus und seinen Verbrechen kann ohne Zweifel behauptet werden, dass diese Phänomene - zumindest auf der kollektiven Ebene - zu einem konstitutiven Teil des deutschen Geschichtsbewusstseins geworden sind. Die Aufarbeitung der Vergangenheit wurde zum „Markenzeichen der politischen Kultur der Bundesrepublik" (Sternfeld 2013: 40f.) und die negative Erinnerung an den Holocaust, die rassistisch motivierte, planmäßige, industriell durchgeführte Vernichtung der Juden, zum zentralen Punkt des nationalen deutschen Geschichtsnarrativs (vgl. Georgi 2009: 92). Nora Sternfeld kann daher in ihrem Buch Kontaktzonen der Geschichtsvermittlung konstatieren: „Heute stellt sich also längst nicht mehr die Frage, ob erinnert wird, sondern wie“ (2013: 125, vgl. Hagemann / Nathanson 2015: 24).

Dieses Wie unterliegt jedoch seit den 1990er-Jahren einem unumkehrbaren Wandel, welcher mit den Begriffen Historisierung, Europäisierung/Globalisierung sowie Pluralisierung der Erinnerung beschrieben werden kann. $\mathrm{Zu}$ dem veränderten Kontext der Geschichtsvermittlung gehören zuerst der wachsende zeitliche Abstand und das Aussterben der Zeitzeugen. Zum anderen ist die Etablierung einer transnationalen Erinnerungskultur zu nennen, markiert durch solche Initiativen wie die Deklaration der Holocaust-Konferenz in Stockholm von 2000 zur Förderung des Holocaust-Gedenkens, -Bildung und -Forschung sowie die Einführung des weltweit am 27. Januar begangenen Holocaustgedenktages (UN-Resolution 2005). Diese transnationale Perspektive auf den Holocaust wird auch innerhalb der staatlichen Grenzen der BRD gewährleistet, denn die deutsche Er- 
innerungsarbeit wird - last but not least - auch mit der Tatsache der ethnischen Pluralisierung der Gesellschaft konfrontiert, welche durch kontinuierliche Einwanderung seit 1955 erfolgt.

Infolgedessen wird der Genozid an den Juden von einem wachsenden Teil der deutschen und europäischen Bürger als weniger zentral in ihrer Geschichte angesehen. Auch sind die Familiengeschichten vieler Migranten gar nicht, bzw. weniger vom Zweiten Weltkrieg und einem kollektiven Schuldgefühl geprägt als es bei den Herkunftsdeutschen der Fall ist. Günther Jikeli sieht die Ursachen dieses Zustands in der Geschichte, Erinnerungskultur und Erinnerungspolitik der Migrationsländer:

Die meisten europäischen Muslim_innen kommen aus den Ländern, die nur eine untergeordnete Rolle im Zweiten Weltkrieg spielten und von denen keine oder relativ wenige Jüdinnen und Juden in deutsche Vernichtungslager in Europa deportiert wurden. In vielen dieser Länder wird jedoch der Holocaust im medialen Diskurs häufig und unwidersprochen in antisemitischer Weise als ein Instrument Israels präsentiert und Holocaust-Leugnungen, Verschwörungstheorien [...] sowie Gleichsetzungen von Israel mit dem nationalsozialistischen Deutschland [also eine Inversion des Opfer-Täter-Verhältnisses] sind keine Seltenheit (Jikeli 2013: 185).

In der Türkei, dem Land, aus dem die mit Abstand größte Einwanderergruppe in der Bundesrepublik kommt, wird der Holocaust zwar als historische Tatsache anerkannt, bildet jedoch keinen Bestandteil der Schulbildung. Als Thema wird er in der Öffentlichkeit nur selten behandelt und gilt als eine vom Establishment instrumentalisierte Vergleichsgröße, um den Genozid an den Armeniern nicht als Völkermord anzuerkennen, während aus den islamistischen Kreisen die Holocaustleugnung, gepaart mit der Kritik an Israel zu vernehmen sind (Bali 2013: $123 \mathrm{f}$.).

Wenn man das bereits Gesagte in Betracht zieht, scheint es auf den ersten Blick allzu selbstverständlich, was Aycan Demirel, der Gründer der Kreuzberger Initiative gegen Antisemitismus feststellt: „Migranten sind in der großen deutschen Erzählung, in der es um die Erinnerung an die NS-Verbrechen und den anspruchsvollen Umgang mit dieser Erinnerung geht, nicht vorgesehen. Nicht als Zuhörer und schon gar nicht als Akteure mit einer eigenen Perspektive" (Topçu / Wefing 2010). Diese Betonung der historischen Schuld als Identitätsmerkmal der Deutschen wird als „ethnisches Paradox“ bezeichnet und als ein Ausschlussmechanismus zunehmend kritisiert. ${ }^{1}$ Folglich wird gefragt, ob es doch nicht eine Alternative dazu gäbe, die Übernahme der fremden Erinnerungen mit ihrer nationalen Perspektive als „Eintrittsbillet“ (Welzer 2003) zur deutschen Nation und seiner Leitkultur zu betrachten, zumal wir es im Allgemeinen auch bei der dritten und vierten Generation der Deutschen von einer weniger auf Schuld, mehr auf

${ }^{1}$ Aleida Assmann zählt Hanno Loewy, Raul Hilberg und Dan Diner als Kritiker der Ethnisierung der Geschichte auf (Assmann 2013: 127). Ähnliche Einwände äußern auch Viola Georgi (2009: 92) und Ulla Kux (2006: 245). 
Verantwortung konzentrierten Erinnerungskultur sprechen können. ${ }^{2}$ Die Tatsache, dass sich immer mehr Migranten auf Grund ihrer historischen Erfahrung, ihrer Familiengeschichte und ihres Kollektivgedächtnisses mit keiner Kategorie der drei von Raul Hilberg entworfenen Seiten der jüdischen Katastrophe, Täter-Opfer-Zuschauer, identifizieren, stellt eine Herausforderung für die Erinnerungs- und Vermittlungsarbeit, besonders in den Medien, Schulen und Gedenkstätten, dar.

Die aktuelle Antwort auf die genannte Herausforderung sind Konzepte, welche monoperspektivistische Identifikationsangbote durch eine Vielfalt von Zugängen, eine Multiperspektivität ersetzen wollen. ${ }^{3}$ Es soll ein transnationaler Verhandlungsraum entstehen, in dem viele Geschichten koexistieren, miteinander in Dialog treten und nicht zuletzt auch miteinander konkurrieren. Noch kommen die größten Immigrantengruppen in Deutschland, mit Ausnahme der Türken, aus den europäischen Ländern, in denen vielfache Bezüge zum Zweiten Weltkrieg und zur NS-Politik bereits bestehen und es ginge darum, diese Zugänge nicht als Alternative zum geltenden deutschen Gedächtnisrahmen, sondern als dessen Ergänzung zu verstehen.

\section{Literarische Zugänge}

Dass die Kunst der Theorie einmal mehr voran ist und diese Multiperspektivität seit Langem leistet, ist eine Binsenwahrheit. Auch die etablierten deutsch-türkischen Autoren wagen die keinesfalls selbstverständliche Beschäftigung mit der negativen deutschen Geschichte, was im Weiteren an ausgewählten Werken gezeigt werden soll. Dabei werden nicht nur die Formen des heutigen Umgangs mit der NS-Zeit und dem Holocaust, sondern auch die aktuellen politischen Diskurse über die Repräsentation und den Umgang mit der (deutschen) Vergangenheit in der Gegenwart reflektiert.

Allen voran ist Zafer Şenocak zu nennen, in dessen Werken, Romanen und Essays, Geschichte und Gedächtnis eine bedeutende Rolle spielen. Im Atlas des tropischen Deutschland fragt er: „Heißt in Deutschland einzuwandern nicht auch, in die jüngste deutsche Vergangenheit einzuwandern?“(1992: 15) und weist darauf hin, dass die Geschichte des Wirkens, der Emanzipation und Assimilierung der Juden als der größten Minderheit anderen Glaubens in Deutschland einen bisher

${ }^{2}$ Dies bestätigt die Studie der Bertelsmann Stiftung Deutschland und Israel heute? von 2013/2014. Sie zeigt, dass die Forderung nach einem Schlussstrich überdurchschnittlich stark von den jüngeren deutschen Befragten vertreten wird: sie wurde von 67 Prozent der Befragten unter 40 Jahren, aber nur 51 Prozent der älteren geäußert (Hagemann / Nathanson 2015: 24 f.).

${ }^{3}$ Zum Beispiel Dan Diners Gegenläufige Gedächtnisse. Über Geltung und Wirkung des Holocaust (2007), Michael Rothbergs Multidirectional Memory: Remembering the Holocaust in the Age of Decolonization (2009), Nora Sternfeld Kontaktzonen der Geschichtsvermittlung. Transnationales Lernen über den Holocaust in der postnazistischen Migrationsgesellschaft (2013). 
noch nicht genug analysierten Erfahrungshintergrund bilde (1992: 15). Im Jubiläumsjahr 1995 sagt der Autor dagegen in einem Interview für Der Tagesspiegel: „Man kann in ein Land einwandern, nicht aber in seine Vergangenheit“" (Şenocak / Yeşilada 1995). Er beklagt sich auch über den ethnisch-kollektiven Charakter des deutschen Gedächtnisses und über die Erinnerungskultur, welche die Geschichte ritualisiert und musealisiert:

Ich habe das Gefühl, diese sogenannte Erinnerungsarbeit hierzulande soll Geschichte einpacken, wie man Gegenstände oder Gebäude einpackt: in Gedenkreden, in Gedenktafeln, in Rituale. Es ist an der Zeit, dass wir die deutsche Geschichte auspacken, daß [sic!] wir Ursachen und Folgen sehen. Erst dann werden wir verstehen, welche Widerstände es heute in Deutschland gegenüber den Einwanderern gibt (Şenocak / Yeşilada 1995).

Diese Aussage enthält die Elemente, die für Şenocaks Beschäftigung mit der deutschen Geschichte charakteristisch sind: die Auseinandersetzung mit dem Komplex der deutschen Schuld und deren Aufarbeitung, die Thematisierung der Ursachen der deutschen Katastrophe und die damit verbundene Aktualisierung der historischen Phänomene durch Vergleich mit der gegenwärtigen Situation.

Auf das Problem der deutschen Schuld kommt Şenocak immer wieder zu sprechen. Deutsche Geschichte ist für ihn geradezu „ein Synonym für Schuld“ (Şenocak 1992: 31). Der Autor würdigt die beispiellose Aufarbeitung der Vergangenheit in der BRD, welche der Schlussstrichpolitik der Nachkriegszeit folgte (Şenocak 2011: 26, 51, 64). Er warnt aber gleichzeitig davor, dass diese „bewältigte" Geschichte durch Ritualisierung zu einer emotionslosen und inhaltlich leeren Metapher des Bösen verkommt, die keinen mehr berührt (Şenocak 1992: 12, 16, 63, 31, Şenocak 2011: 116). Er fragt: „Wie kann »Auschwitz« von der Gedenkstätte und dem sprachlichen Allgemeinplatz, den es heute darstellt, wieder in jenen Ort zurückverwandelt werden, der das Gelände des Schreckens repräsentiert, die Erinnerung an den von einem verbrecherischen Regime an Tausenden von Menschen verübten Mord?"“ (1992: 60)

Eine ähnliche Bewertung der deutschen Erinnerungskultur wird in Şenocaks Roman Gefährliche Verwandtschaften präsentiert. Die Betroffenheit als Basis der Versöhnung zwischen Opfern und Tätern wird als von Gruppen nachgestellte Trauer, eine Trauer „nach dem Kalender“ kritisiert. In dem Roman ist das Problem der Schuld sogar verdoppelt. Die in Berlin lebende Hauptfigur des Romans, Sascha Muchteschem, ist Sohn einer deutschen Jüdin und eines Türken. Seine Großeltern mütterlicherseits haben sich durch die Flucht in die Türkei vor dem tragischen Schicksal ihrer Verwandten retten können. In den 1950er-Jahren kehrten sie nach Westdeutschland zurück, ohne die Erinnerung an den Holocaust an ihren Enkel zu vermitteln. Der verweigerte Zugang zu der Familiengeschichte, ihre Tabuisierung löst in Sascha einen Sammlerfleiß und eine Faszination für die Dokumente der NS-Zeit aus, welche er bei dankbaren, oft betagten Hitleranhängern aufspürt.

Nach dem plötzlichen Tod seiner Eltern erbt Sascha ein Tagebuch des Großvaters väterlicherseits, das ein Geheimnis enthält. In einem mühseligen Entziffe- 
rungsprozess erfährt der Enkel, dass der türkische Großvater im staatlichen Dienst im Jahre 1915 an den Deportationen der Armenier beteiligt war, was ihn bis zu seinem unaufgeklärten Selbstmord als Mitglied der türkischen Delegation für die Olympischen Spiele in Berlin 1936 belastete. Sascha, der auf keine Erinnerungen seiner Eltern, auf kein kommunikatives Gedächtnis, wie es Jan Assmann definiert hat (Assmann 2007: 50f.), zurückgreifen kann, ist somit ein Erbe sowohl der Opfer als auch der Täter, fühlt sich aber keiner dieser Gruppen angehörig.

Es stellt sich natürlich die Frage, was Şenocak mit diesem sich dem Lesen aufdrängenden Vergleich beider Genozide bezweckt. Der Autor selbst wehrt sich gegen den Vorwurf einer Gleichsetzung. Zu der Interpretation des Romans erklärte Zafer Şenocak: er wollte „das Beredte, dieses Darübersprechende, Es-wieder-insZentrum-Rückende der deutschen Gesellschaft, Holocaust als Leitthema der deutschen Gegenwart, und dieses absolute Schweigen, dieses Verknoten im Türkischen“ gegenüberstellen (Cheesman 2003: 27).

Im Roman Gefährliche Verwandtschaft gibt es viele Stellen, wo Schicksale von Juden, Türken und Armeniern von gestern und heute in Verbindung gebracht werden (u.a. wird die Aufnahme von jüdischen Flüchtlingen in der Türkei nach Hitlers Machtübernahme thematisiert). Der Autor konzentriert sich aber nicht auf die Geschichte der Vernichtung, sondern auf die Prozesse der Verfremdung und Ausgrenzung von Gruppen, die im Zuge der Nationsbildung auf ihre Ethnie oder Religion reduziert und als solche von der Mehrheitsgesellschaft ausgegrenzt wurden. Solche Phänomene der Ausgrenzung bilden auch den Hintergrund der Gegenwartshandlung des Romans, welche sich im Nachwende-Berlin, Anfang 1990er-Jahre abspielt. In dieser Zeit wird nach Şenocak die nationale Identität zum wichtigen Diskussionspunkt: „Wenn aber heute die Frage gestellt wird, wer ein Deutscher ist und wer nicht, schaut man auf die Türken. An ihnen werden die Grenzen des Deutschseins getestet. Juden, die sich über ihr Deutschsein klar werden wollen, entdecken im Spiegel die Türken.“(2009: 90).

Ähnlichen Entfremdungsmechanismen unterliegt auch der Protagonist des späteren Romans von Zafer Şenocak Alman Terbiyesi (2007). Deutsche Schule, so der Titel der deutschen Übersetzung aus dem Jahre 2012, erzählt die Geschichte von Salih Süvari, der während des Zweiten Weltkriegs, genau in der Zeit vom Barbarossa-Feldzug bis zur Schlacht um Stalingrad in Istanbul seine Memoiren schreibt. Salih stammt vom Balkan. Er ging gegen 1900 nach Deutschland, um sich dort zum Offizier ausbilden zu lassen. Die nächsten vierzig Jahre blieb er in Berlin, zuerst als Soldat, dann als Geschäftsmann. Dort übernahm er die nationalistischen Ideen der Zeit. Er wurde zum deutschen Nationalisten, der gleichzeitig auch türkische Interessen vertrat und diese doppelte Zugehörigkeit als Vorteil sah: „Was für ein Privileg, sowohl Türke als auch Deutscher zu sein. Zwei freien Völkern zugleich angehören, die im selben Jahrhundert das Zeug dazu hatten, aus ihrem ureigenen Willen die Geschichte [zu] lenken" (Şenocak 2012: 19). Salih bewundert die Deutschen wegen ihres technologischen Vorsprungs, ihrer Kultur, 
ihrer Sekundärtugenden und vor allem wegen ihres Militärs. Er ist kein Freund der Demokratie und gegen Hitler weiß er nichts einzuwenden, außer dass er mit dem Angriff auf Moskau einen strategischen Fehler machte (2012: 83). Den Völkermord an Armeniern, der im Romantext kurz angesprochen wird, lobt Salih als „mutig und historisch“ (2012: 75). Dass dabei „ein paar gestorben sind“, hält er für „natürlich“ (2012: 75). Hitlers Politik gegen die Juden lässt ihn kalt, was sich von seiner deutschen Frau nicht behaupten lässt. Im Sommer 1939 veranlasst sie aus Protest den Umzug nach Istanbul.

Das Problem der Judenverfolgung holt Salih jedoch Anfang der 1940er-Jahre in Person seiner ehemaligen Geliebten, Karla, ein. Auf der Flucht nach Palästina besucht sie ihn in Istanbul und bittet um Hilfe, die er mit Selbstverständigkeit leistet. Im Gespräch mit der verzweifelten gehetzten Frau, ehemals kosmopolitisch veranlagten Modeschöpferin, regt sich in Salih Zweifel auf. Karla beschuldigt ganz Europa sich an der Judenverfolgung schuldig gemacht zu haben: „Jeder, der stillhält und nur zusieht, ist schuldig“ (2012: 104). Sie fordert Salih auf, Stellung zu nehmen.

An den späteren Einträgen des Tagebuchs merkt man, dass Karlas Appel Wirkung zeigt. Eine Schilderung des Treffens Salihs mit amerikanischem Geschäftsmann und Freund, das in Berlin am Tag der Machtübernahme von Hitler stattfand, endet mit der kritischen Beurteilung der eigenen Untätigkeit. Dem Plädoyer des Amerikaners gegen die vermeintliche Weltherrschaft der Juden wagte der Türke nicht zu widersprechen. Dafür findet er folgende Erklärung: „Bestätigt man uns in unserer Ansicht, so fühlen wir uns nicht bemüßigt, Fragen zu stellen, Zweifel zu hegen. Allein die Bestätigung stimmt uns zufrieden“" (2012: 121).

Im Fokus der Geschichte steht im Roman weder der Täter noch das Opfer, sondern der Zuschauer, dessen Passivität es den Tätern erlaubt, andere zu Opfern zu machen. Diese Passivität charakterisiert im Roman zuerst die zentrale Gestalt. Salih ist vom NS-Regime teilweise authentisch fasziniert und verspricht sich von Hitlers Politik bestimmte Vorteile für seine alte Heimat, teilweise resultiert seine Haltung aus Angst vor Verratsvorwurf (2012: 38) sowie aus dem preußischen Ethos der Offiziersehre. Aber auch die Türkei wird im Roman im Zwielicht des Lavierens gezeigt, nach dem Motto der Regierung „Deutschland ist unser Freund, England unser Verbündeter" (2012: 86). Im Roman wird nicht nur an die Aufnahme der jüdischen Flüchtlinge aus dem nationalsozialistischem Deutschland erinnert, sondern auch an das Schicksal der Struma (2012: 123). Im Februar 1942 verweigerte die neutrale Türkei den fast 800 jüdischen Flüchtlingen aus Rumänien einen Landgang und schleppte nach wochelangem Warten das fahruntüchtige, überfüllte Schiff aufs Schwarze Meer zurück. Am nächsten Tag wurde es von einem sowjetischen U-Boot versenkt.

Die Unterlassung kann hier als Schuld gedeutet werden, doch die Anteilnahme hat auch ihren Preis. Salih Süvari, durch seine hybride Identität ohnehin beiden Seiten, Deutschen wie Türken verdächtig, wird nach Karlas Besuch von Berlin als ein mit Juden verkehrender Mann eingestuft und zur persona non grata erklärt 
(2012: 136). Mit dem Entzug der deutschen Staatsbürgerschaft ist sein Ausschluss aus der deutschen Volksgemeinschaft symbolisch vollzogen.

Im Roman Selam Berlin (2003) der deutsch-türkischen Autorin Yadé Kara bildet die Zeit des Nationalsozialismus keine Geschichtskulisse mehr. An einigen Stellen dient sie jedoch als Referenzpunkt für die Entwicklungen in Berlin zwischen dem Mauerfall und der deutschen Vereinigung. Kara schildert, wie parallel zu dem Fortschreiten des deutschen Vereinigungsprozesses die Nationsbildung auf der Grundlage der Blutsgemeinschaft fortschreitet und die Tendenz zur Abgrenzung gegenüber Fremden wächst, die gegen Ende des Romans in Gewalt der Neonazis gegen Ausländer mündet.

Wie die bereits erwähnten Figuren von Şenocak ist der Protagonist in Selam Berlin, der neunzehnjährige Hasan Kazan, ein Mann, der in mehreren Kulturen zu Hause ist. Wenn es unbedingt sein muss, identifiziert er sich am meisten lokal mit der Stadt Berlin. Als Mitglied einer sichtbaren Minderheit wird er und seine Freunde zum Ziel von gewalttätigen Angriffen. Um seine Gefühle in der Situation der direkten Bedrohung zu beschreiben, bedient sich Hassan des historischen Exempels:

Mir fiel die Frau ein, über die ich mal gelesen hatte, daß [sic!] sie mit ihrer Familie die ganze Nacht von einer Endstation zur anderen U-Bahn gefahren war, um in der Pogromnacht nicht aufzufallen. [...] Aber ich war nicht blond, wie die Frau. Ich war dunkel und fiel auf. Hier wurde ich wegen meiner schwarzen Haare und braunen Augen angegriffen und nicht wie in Istanbul wegen meiner Brieftasche (Kara 2003: 347).

Der Rassismus, dem Hasan in Berlin mit steigender Häufigkeit begegnet, bedient sich auch einer undifferenzierten Sprache, in der Fremdbilder austauschbar zu sein scheinen. Beschimpfungen wie „Kanackensau und Judenpack“ (Kara 2003: 346) werden in einem Atemzug genannt. Als Hasan eine Kneipe besucht, wo er im Endeffekt nicht bedient wird, muss er sich folgende Szene anschauen: ,»Det is een Jude«, sagte ein zahnloser Greis zu seinem Nachbarn. »Vielleicht isa jar keener«, erwiderte sein Nachbar mit Glatze. »Dann is er een Türke - das kommt aufs jleiche raus" (2003: 232). Erlebnisse dieser Art bringen Hasan am Ende des Romans zu der Entscheidung, Berlin zu verlassen. Seine Cousine Leyla, die Tochter eines Türken und einer Deutschen, distanziert sich verbal von Deutschland und seiner Vergangenheit. In die Rolle des Opfers gedrängt, ist sie nicht bereit, die Verantwortung für die Tätergeschichte zu übernehmen: „Hermann Hesse, Hitler, Holocaust... Ich will mit diesem ganzen Schrott nichts zu tun haben. Ich will alles aus meinem Kopf wischen...“ (2003: 168).

Die Austauschbarkeit der Türken und Juden funktioniert im Roman auch andersrum. Ein bekannter Regisseur, für den Hasan die Rolle eines türkischen Drogendealers spielte, stellt fest, dass die Türkenthematik schon ,ausgelutscht“ sei und das Publikum nicht mehr ,hammelschlachtende Väter und Gemüsebrüder“ sehen will (2003: 373) und entdeckt die Ostjuden als Thema für seinen neuen Kinohit. Eine andere Variante der Neuentdeckung der Juden, ist das Interesse der Westdeutschen an den Spuren des früheren jüdischen Lebens in Ostberlin. Wie 
die Islamfeindlichkeit und der Antisemitismus parallelisiert werden, so werden es auch der Orientalismus und der Philosemitismus. Der gemeinsame Nenner für diese Phänomene ist eine Haltung gegenüber Fremden, welche Menschen essentialistisch auf wenige Eigenschaften reduziert und diese als wesensfremd darstellt.

Vergleiche von Türken und Juden werden noch expliziter in Feridun Zaimoglus Werk Koppstoff. Kanaka Sprak vom Rande der Gesellschaft artikuliert. Es enthält eine Reihe von fiktiven Protokollen der Interviews mit jungen Frauen mit türkischem Migrationshintergrund. Im Vorwort wird der soziohistorische Kontext der Entstehung von Koppstoff (1998) erörtert: Es ist Deutschland Ende der 1990er-Jahre, wo „rechtsradikale Gewalt, verbal wie körperlich, eine Alltagserscheinung wie der Gang zum Bäcker [ist]. Proteste sind ob der Gewöhnung selten geworden. [...] Die Stichworte kommen längst aus der Mitte der Gesellschaft" (Zaimoglu 2011: 122). Vor diesem Hintergrund nehmen die im Buch porträtierten Frauen in verschiedenen Variationen vergleichend Bezug auf die Judenverfolgung und den Holocaust. So sagt Beispielsweise Nazan: die Kanaken seien „so was wie Judenschneider im geflickten Rock“ (2011: 137). Devrim, eine Studentin, bezeichnet sich selbst als „das geborene Opfer“, und behauptet von den deutschen Kindern, sie würden strahlen, als hätten sie soeben ein paar Juden in den Ofen geschoben, während im Ghetto „das Kanak-Kid nach more future“ brülle (2011: 209). Sie kritisiert die Schlussstrichforderungen der Deutschen (2011: 210). Beiläufige antisemitische Sprüche der „Arischsau“ (2011: 213, 221) und das „Schädelmessungsgewerbe" (2011: 207) finden in den Interviews Erwähnung.

Die Verbindung von Vergangenheit und Gegenwart eines Einwanderungslandes ist für die Thematisierung des Holocausts in der Literatur der deutsch-türkischen Autoren zentral. Viola Georgi, die das Verhältnis der Migrantenjugendlichen zum Nationalsozialismus und dem Holocaust untersuchte, hat einige Idealtypen der Geschichtsbezüge erarbeitet. In den genannten Beispielen der literarischen Figuren scheint der erste Typus am häufigsten vertreten zu sein: Charakteristisch ist für ihn die „Verhandlung des historischen Opferstatus über die Strategie von Analogiebildungen. Selbsterlebte Diskriminierung und Rassismus in der deutschen Mehrheitsgesellschaft werden zu den Ausgrenzungs- und Verfolgungsmechanismen des NS-Regimes in Beziehung gesetzt" (Georgi 2009: 103). Aleida Assman bezeichnet eine solche Verknüpfung unterschiedlicher historischer Leiderfahrungen ohne Löschung der Differenz dieser Geschichten als opferidentifizierte Erinnerung (Assman 2013: 129). Diese Praxis kann man als eine gefährliche Verengung der Perspektive verstehen, die auf Entkontextualisierung, Enthistorisierung und Relativierung des Holocausts hinausläuft. Man kann sie aber auch wie Alexandra Lübcke als eine Art „Inter- bzw. Transnationalisierung des Holocaust" deuten, eine „Verknüpfung von lokalen und nationalen hin zu transnationalen Erinnerungs- und Erfahrungsräumen“ (Lübcke 2009: 84).

In Şenocaks Romanen ist auch Georgis zweiter Typus der Geschichtskonstruktion anzutreffen, nämlich die „Bezugnahme auf die Zuschauer, Mitläufer und Tätergesellschaft, deren Sozialperspektiven probeweise angenommen werden“ 
(Georgi 2009: 103). Diese Perspektive scheint mir im deutschen Erinnerungsdiskurs besonders interessant zu sein. Denn sie berührt eine Frage, welche Peter Reichel in Deutschen Erinnerungsorten (2001) in seinem Artikel über Auschwitz, trotz der Globalisierung der Erinnerung über diesen Ort, als eine für den deutschen Charakter dieses Ortes grundlegende Frage versteht: Warum Hitler nicht verhindert werden konnte und warum das Gewaltverbrechen gerade in Deutschland geschehen ist (Reichel 2009: 621).

Im Weiteren sollen einige nichtliterarische Beispiele der Auseinandersetzung mit der deutschen NS-Vergangenheit kurz vorgestellt werden, die sowohl die Opfer- als auch die Täter- und Mitläuferperspektive berücksichtigen.

\section{Nichtliterarische Zugänge}

Im Bereich der performativen Kunst muss der Schauspieler, Regisseur und Musiker Serdar Somuncu erwähnt werden, der in einer Kombination aus Lesung, Theater und Kabarett die in Texten überlieferte deutsche Vergangenheit aufs Korn nimmt. Im Jahre 1996 ging Somuncu auf Tournee, um aus Hitlers Mein Kampf vorzulesen und das Vorgelesene zu kommentieren und lenkte damit die Aufmerksamkeit vor allem der Medien und der Neonazis auf sich. In über 1400 Vorstellungen in Deutschland, Österreich, Dänemark, Holland, Liechtenstein und Tschechischer Republik betrieb er jahrelang eine „neue Form der Geschichtsaufklärung“ (Kas / Marunic 2015) und gleichzeitig einen Tabubruch. Somuncu las Fragmente aus dem Text, indem er sich schauspielerisch in Hitler versetzte, unterbrach diese durch eigene Kommentare und Anekdoten von den Lesungen.

Sein Ziel war eine Entmythologisierung des Textes, dem eine Aura des Verbotenen anhaftete. Durch den schonungslosen Umgang mit dem kanonischen Text des Nationalsozialismus, die Aufdeckung der Banalität und Absurdität mancher darin enthaltenen Bilder und Formulierungen, wurde Hitlers Werk der Lächerlichkeit preisgegeben.

Natürlich war Somuncu nicht der Erste, der Hitler mit Mittel des Humors und der Groteske bloßzustellen versuchte. Seine großen Vorgänger waren unter anderem Charles Chaplins The Great Dictator (1940), Bertold Brechts Drama Der aufhaltsame Aufstieg des Arturo Ui (1941), das Somuncu vor seinen Lesungen inszenierte, Ernst Lubitsch' Film Sein oder Nichtsein (1942), Mel Brooks The Producers/Frühling für Hitler (1968), George Taboris später verfilmtes Stück Mein Kampf (1987) und der erste Teil von Christoph Schlingensiefs Film 100 Jahre Adolf Hitler-Die letzte Stunde im Führerbunker (1989). ${ }^{4}$ Eine direkte Inspiration und das Vorbild war jedoch für Somuncu der österreichische Schauspieler Helmut

${ }^{4}$ Die Reihe von Komödien über Hitler wurde in der letzten Zeit mit Filmen von Dani Levy Mein Führer - Die wirklich wahrste Wahrheit über Adolf Hitler (2007) und David Wnendt Er ist wieder da (2015) fortgesetzt. 
Qualtinger, der in den 1970er-Jahren ebenfalls humoristische szenische Lesungen von Mein Kampf veranstaltete (Boran 2004: 264).

Somuncu wollte aber Mein Kampf nicht einfach verspotten und damit das Publikum unterhalten, sondern strebte gleichzeitig eine inhaltliche Auseinandersetzung mit der Ideologie an, die sich, wie man weiß, als verführerisch und äußerst gefährlich erwies. Da sich Hitlers Ziele, die Vernichtung der Juden und der Krieg um mehr Lebensraum, deutlich an seinem Text erkennen lassen, stellte Somuncu die Frage nach der Anfälligkeit des Durchschnittsmenschen für rassistisches Gedankengut. Die Aktualität dieser Frage sprach und spricht der Künstler immer wieder in seinen Auftritten an. In einem Interview gab er als ausschlaggebenden Anlass zu seinen Mein Kampf-Lesungen die ausländerfeindlichen Ausschreitungen gegen Türken und Asylbewerber Anfang der 1990er-Jahre an (Kas / Marunic 2015).

Wie bereits gesagt, war Somuncu nicht der Erste, der den deutschen Diktator auf der Bühne verspottete, er war aber der erste Türke. Als Vertreter der größten Einwanderergruppe in der BRD hatte er selbstverständlich die Legitimation, das Thema der Lesung immer wieder zu aktualisieren und auf die gegenwärtige Ausländerfeindlichkeit, Rassismus und Diskriminierung Bezug zu nehmen. Sein Recht auf Auseinandersetzung mit der NS-Vergangenheit musste er erst bestreiten:

Auch wenn wir Türken keinen Großvater haben, der in der NSDAP war, auch wenn wir niemanden in der Verwandtschaft haben, der eine braune Vergangenheit hat, so sind wir doch mitverantwortlich für die Aufarbeitung der deutschen Thematik, weil wir keine gemeinsame Gegenwart und Zukunft verlangen dürfen, ohne einen Besitzanspruch auf die Bewältigung der deutschen Vergangenheit zu stellen (Somuncu 2002: 173).

Die Erlebnisse von seiner Tour bearbeitete Somuncu in dem Tagebuch Nachlass eines Massenmörders - Auf Lesereise mit „, Mein Kampf“" (2002). Seine „Aufklärungsmission“" setzte er mit ähnlich gestalteten Lesungen aus Joseph Goebbels' Sportpalastrede Wollt ihr den totalen Krieg? vom Februar 1943 fort. Somit ging er noch weiter als Şenocak und wandte sich den Tätern zu, nahm wortwörtlich deren Perspektive an, um sie immer wieder zu reflektieren, zu dekonstruieren und im aktuellen Kontext zu beleuchten.

Die Aufgabe der Aufarbeitung der schwierigen Vergangenheit, die für alle in Deutschland Lebenden gelten soll, was Serdar Somuncu in der oben zitierten Aussage betonte, spielt auch im Film Der letzte Mentsch unter der Regie von Pierre-Henry Salfati eine wichtige Rolle. Die französisch-deutsch-schweizerische Produktion aus dem Jahre 2014 handelt von einer Reise des in Köln lebenden Marcus Schwarz in seine jüdische Vergangenheit. Marcus, eigentlich Menachem Titelbaum, ist ein Holocaustüberlebender, der seine Identität beweisen muss, um nach dem Tod auf einem jüdischen Friedhof ruhen zu können. Auf seiner Tour durch Europa begleitet ihn eine junge Frau, die 20-jährige Gül, deren Eltern aus der Türkei kommen.

Der letzte Mentsch ist primär die Geschichte der erzwungenen Auseinandersetzung eines alten Mannes mit seinem tragischen Schicksal. Der Vergangenheit 
Titelbaums muss sich aber auch seine Reisegefährtin Gül stellen, was zu ihrer Verwandlung führt. Am Anfang ist die Frau eine Banausin, welche glaubt, dass man das Jüdischsein durch einen Blutstest nachweisen kann und unreflektiv die Aussage eines „komischen Türkeiforschers“ wiederholt, dass Türken die neuen Juden Europas seien, heute in Deutschland genauso unbeliebt wie damals die Juden. Schwarz protestiert gegen einen solchen undifferenzierten Vergleich mit dem Hinweis darauf, dass die Nazis Millionen von Juden umgebracht haben.

Im Laufe der Handlung begreift Gül nicht nur die Dimension der Vernichtung, sondern auch ihre menschenverachtende Natur, mit der sie durch die exemplarische Geschichte der Familie Titelbaum konfrontiert wird. Sie überredet Schwarz, seine Erinnerungen auf Video für die Shoah Foundation aufzunehmen, um sie nachfolgenden Generationen zugänglich zu machen. Schließlich lässt sie sich die Lagernummer des Mannes tätowieren, wie dies neulich die Enkel der Holocaustüberlebenden in Israel tun. Die Zukunft der Erinnerung an die NS-Vergangenheit, welche mit dem Aussterben der Augenzeugen nicht enden darf, so scheint der Film zu suggerieren, hat auch das Gesicht von Gül, einer Wahldeutschen, die ihren eigenen Zugang zu der Geschichte gefunden hat.

Wie unterschiedlich die Wege der Einwanderer zu der deutschen Geschichte und wie erfolgreich ihr Lernprozess in Wirklichkeit sein können, zeigt ein Projekt unter dem Titel Stadtteilmütter auf den Spuren der nationalsozialistischen Geschichte. Stadtteilmütter sind bildungsbewusste Frauen mit Migrationshintergrund, die in sozialen Brennpunkten leben und als Familienberaterinnen arbeiten. Das in Kooperation vom Diakonischen Werk und Aktion Sühnezeichen Friedensdienste (ASF) entworfene Programm umfasst Seminare zur nationalsozialistischen Geschichte. Sie schließen gemeinsame Sitzungen, Besichtigungen von Orten, die mit dem Nationalsozialismus verbunden waren und an ihn erinnern sowie Gespräche mit Zeitzeugen ein. Die Präsentation der individuellen Migrationsgeschichte der Frauen ist ebenso ein Bestandteil der Seminarreihe.

Das Projekt, das seit 2006 fortgesetzt wird, ist auf den ausdrücklichen Wunsch der Stadtteilmütter entstanden. Jutta Weduwen, die das Programm seitens der ASF betreut, erklärt die Motive der Migrantinnen, sich mit der NS-Vergangenheit zu beschäftigen, wie folgt:

Sie wollten verstehen, wie der Nationalsozialismus als politisches und gesellschaftliches System funktionieren konnte, ob und wo es Kontinuitäten in der aktuellen deutschen Gesellschaft gibt und wo die Geschichte noch heute sichtbar und spürbar ist. Die Teilnehmerinnen der Seminarreihe hatten ein großes Interesse daran, sich generell mit den Mechanismen von Ausgrenzung, Verfolgung und Völkermord zu beschäftigen. [...] Die Stadtteilmütter wollten ihren Kindern Antworten geben können und sich mit ihnen über dieses wichtige zeitgeschichtliche Thema austauschen. Für einige Frauen haben der Nationalsozialismus und der Zweite Weltkrieg zudem eine wichtige Bedeutung für die Beziehung ihres Herkunftslandes zu Deutschland (ASF 2010: 8 f.).

Der ersten Gruppe der Stadtteilmütter wurde gleich nach dem Abschluss der Seminarreihe klar, dass sie die Ergebnisse des dialogischen Lernprozesses nicht 
nur für sich allein behalten können, sondern als Multiplikatorinnen für andere Migrantinnen und andere Teile der deutschen Gesellschaft wirken wollen. Aus dieser inneren Überzeugung, die Erfahrungen und das Wissen teilen zu müssen, sind öffentliche Präsentationen, Stadtteilführungen und eine Dokumentationsbroschüre unter dem Titel Unsere Geschichten - eure Geschichte? entstanden, in der vierzehn Frauen aus Berlin Kreuzberg und Neukölln (mit Wurzeln in der Türkei, Sri Lanka, Kurdistan-Irak, Polen, Eritrea und dem Kosovo) über ihre sehr emotionale und manchmal erschütternde Begegnungen mit der deutschen Vergangenheit sowie ihre eigenen Lebensgeschichten erzählen. Drei Teilnehmerinnen des Programms wurden auch von Julia Oelkers in einem 30-minütigen Dokumentarfilm Es ist auch meine Geschichte - Stadtteilmütter auf den Spuren des Nationalsozialismus bei Treffen mit Zeitzeugen und Nachgeborenen porträtiert. Die Botschaft, die darin verkündet wird, ist klar: die Biographie und Herkunft der Migranten beeinflusst ihre Sicht auf die deutsche Geschichte, aber sie versperrt nicht den Zugang zu ihr.

Auch wenn man neben den oben kurz geschilderten Projekten noch Esther Bejarano nennt, die inzwischen über 90-jährige ehemalige Akkordeonistin im Mädchenorchester des KZ-Auschwitz, die seit 2009 mit der Kölner Hip-Hop-Gruppe, der Microphone Mafia (Kutlu Yurtseven und Rossi Pennino, beide Gastarbeiterkinder) auf Tour ist, um aus ihrer Biografie zu lesen und die Musik als Mittel zur Auseinandersetzung mit der Vergangenheit und gegen rassistische und rechtsextreme Tendenzen in der Gegenwart zu benutzen (Wagner 2016), ${ }^{5}$ wird das im Titel dieses Artikels umrissene Thema nicht ausgeschöpft sein.

Die Beschäftigung mit der nationalsozialistischen Vergangenheit als „Eintrittsbillet"? Ja, aber unter wichtigen Bedingungen: der multiperspektivische Umgang und der Gegenwartsbezug (nicht als Ziel, sondern als Voraussetzung) müssen akzeptiert und eine Identifikation mit dem deutschen Muster der Betroffenheit darf nicht verlangt werden.

\section{Literatur}

Aktion Sühnezeichen Friedensdienste e.V. (Hrsg.) (2010): Unsere Geschichten - eure Geschichte? Neuköllner Stadtteilmütter und ihre Auseinandersetzung mit der Geschichte des Nationalsozialismus. Ein Projekt von Aktion Sühnezeichen Friedensdienste. Berlin.

Assmann, Aleida (2013): Das neue Unbehagen an der Erinnerungskultur. Eine Intervention. München.

Assmann, Jan (2007): Das kulturelle Gedächtnis. Schrift, Erinnerung und politische Identität in frühen Hochkulturen. 6. Aufl. München.

${ }^{5}$ Auch über dieses Projekt entstand ein Dokumentarfilm Film ab! Gegen Nazis (Regie: Katharina Obens / Tanja Seider, 2010, 60 Min.) 
Bali, Rifat N. (2013): Die Wahrnehmung des Holocaust in der Türkei. In: Jikeli, Günther / Stoller, Kim Robin / Allouche-Benayoun, Joëlle (Hrsg.): Umstrittene Geschichte. Ansichten zum Holocaust unter Muslimen im internationalen Vergleich. Frankfurt am Main. S. 123-134.

Boran, Erol M. (2004): Eine Geschichte des türkisch-deutschen Theaters und Kabaretts. Diss. Universität Ohio.

Cheesman, Tom (2003): „Einfach eine neue Form “: Gespräch mit Zafer Şenocak. In: Cheesman, Tom / Yeşilada, Karin (Hrsg.): Zafer Şenocak. Cardiff. S. 19-30.

Der letzte Mentsch (2014). Reg. Pierre-Henry Salfati. Deutschland/Schweiz/Frankreich. DVD Farbfilm Verleih Berlin, ca. 99 Min.

Es ist auch meine Geschichte - Stadtteilmütter auf den Spuren des Nationalsozialismus. (2011). Reg. Julia Oelkers. Deutschland ASF, ca. 30 Min.

Georgi, Viola B. (2009): „Ich kann mich für Dinge interessieren, für die sich jugendliche Deutsche auch interessieren ". Zur Bedeutung der NS-Geschichte und des Holocaust für Jugendliche aus Einwandererfamilien. In: Georgi, Viola B. / Ohliger, Rainer (Hrsg.): Crossover Geschichte. Historisches Bewusstsein Jugendlicher in der Einwanderungsgesellschaft. Hamburg. S. 90-108.

Hagemann, Steffen / Nathanson, Roby (2015): Deutschland und Israel heute. Verbindende Vergangenheit, trennende Gegenwart? Gütersloh, https://www.bertelsmann-stiftung.de/de/publikationen/publikation/did/deutschland-und-israel-heute/ (Zugriff am 28.09.2016).

Jikeli, Günther (2013): Wahrnehmungen des Holocaust unter jungen Muslimen in Berlin, Paris und London. In: Jikeli, Günther / Stoller, Kim Robin / Allouche-Benayoun, Joëlle (Hrsg.): Umstrittene Geschichte. Ansichten zum Holocaust unter Muslimen im internationalen Vergleich. Frankfurt am Main. S. 187-226.

Kara, Yadé (2013): Selam Berlin. Zürich.

Kas, Onur / Marunic, Dajana: Der Türke, der „,Mein Kampf“ liest, http://www.dasbiber.at/content/ der-tuerke-der-mein-kampf-liest (Zugriff am 28.09.2016).

Kux, Ulla (2006): Deutsche Geschichte und Erinnerung in der multiethnischen und -religiösen Gesellschaft. Perspektiven auf interkulturelle historisch-politische Bildung. In: Behrens, Heidi / Motte, Jan (Hrsg.): Politische Bildung in der Einwanderungsgesellschaft. Schwalbach/Taunus. S. 241-259.

Lübcke, Alexandra (2009): Enträumlichungen und Erinnerungstopographien: Transnationale und deutschsprachige Literaturen als historiographisches Erzählen. In: Schmitz, Helmut (Hrsg.): Von der nationalen zur internationalen Literatur. Transkulturelle deutschsprachige Literatur und Kultur im Zeitalter globaler Migration. Amsterdam/New York. S. 77-97.

Reichel, Peter (2009): Auschwitz. In: François, Etienne / Hagen, Schulze (Hrsg.): Deutsche Erinnerungsorte. Bd. 1. München. S. 600-621.

Şenocak, Zafer / Yeşilada, Karin (1995): Darf man Türken mit Juden vergleichen, Herr Şenocak? In: Der Tagesspiegel, 13./14.04.

Şenocak, Zafer (1992): Atlas des tropischen Deutschland. Berlin.

Şenocak, Zafer (2012): Deutsche Schule. Roman. Berlin.

Şenocak, Zafer (2011): Deutschsein. Eine Aufklärungsschrift. Hamburg.

Şenocak, Zafer (2009): Gefährliche Verwandtschaft. 2. Aufl. München.

Somunçu, Serdar (2002): Nachlass eines Massenmörders. Auf Lesereise mit „Mein Kampf“. Bergisch Gladbach.

Topçu, Özlem / Wefing, Heinrich (2010): „Bist du Jude? “ Zwei Deutschtürken versuchen, die deutsche Geschichte zu erklären - und treffen auf hartnäckige Vorurteile. In: Die Zeit 21.01, http:// www.zeit.de/2010/04/Umfrage-Reportage?page=all (Zugriff am 28.09.2016).

Wagner, Dirk (2016): Die Auschwitz-Überlebende, die gegen rechts rappt. In: Süddeutsche Zeitung 08.03, http://www.sueddeutsche.de/bayern/regensburg-rap-gegen-rechts-1.2894445 (Zugriff am 29.09.2016). 
Welzer, Harald (2003): Deutsches Eintrittsbillet. Was die Kinder von Einwanderern über den Holocaust denken. In: Süddeutsche Zeitung 22.10.

Zaimoglu, Feridun (2011): Kanak Sprak / Koppstoff. Die gesammelten Mißtöne vom Rande der Gesellschaft. Köln.

\section{Abstracts}

Die Erinnerung an den Nationalsozialismus und den Holocaust bleibt in Deutschland ein negativer Referenzpunkt der nationalen Identität. Mit der Verwandlung der Bundesrepublik in ein Einwanderungsland wird jedoch immer häufiger gefragt, inwieweit dieses genealogische, ethnisierte Modell des Erinnerns seine Gültigkeit behalten kann und soll. Der Beitrag geht den Fragen nach, in welchem Maße und auf welche Weise die Vertreter und Nachfahren der türkischen Einwanderer in der BRD die NS-Vergangenheit in ihren literarischen Werken und anderen Projekten thematisieren und wie sie zum Problem der historischen deutschen Schuld stehen.

Schlüsselwörter: kulturelles Gedächtnis, Nationalsozialismus, Holocaust, deutsch-türkische Autoren

\section{The "entrance card" into the German society? The National Socialist past from the German-Turkish perspective}

The memory of the National Socialism and the Holocaust remains a common negative reference point for the national identity in the FRG. At the same time, Germany's transformation into an immigration country raises the question, to what extent this ethnicised genealogical concept of remembering can or should apply to the whole society. The paper examines literary works and projects of Turkish immigrants in Germany and their descendants to show how they address matters related to the Nazi past and reflect on the issue of German guilt.

Keywords: cultural memory, National Socialism, Holocaust, German-Turkish authors

Dorota Masiakowska-Osses

Uniwersytet im. Adama Mickiewicza w Poznaniu

Instytut Filologii Germańskiej

Al. Niepodległości 4

61-874 Poznań

Polen

E-Mail: domaska@amu.edu.pl 